Supporting Information of

\title{
Handling Ensemble $N$-Representability Constraint in Explicit-by-Implicit Manner
}

\author{
Yi-Fan Yao ${ }^{1}$, Wei-Hai Fang ${ }^{1,2}$ and Neil Qiang $\mathrm{Su}^{*, 1}$ \\ ${ }^{1}$ Department of Chemistry, Key Laboratory of Advanced Energy Materials Chemistry (Ministry of \\ Education) and Renewable Energy Conversion and Storage Center (RECAST), Nankai University, \\ Tianjin 300071, China \\ ${ }^{2}$ Key Laboratory of Theoretical and Computational Photochemistry, Ministry of Education, College \\ of Chemistry, Beijing Normal University, Beijing 100875, China
}

*E-mail: nqsu@nankai.edu.cn

(Dated: July 14, 2021) 


\section{The Electronic Energy in RDMFT}

The electronic energy in $\mathrm{RDMFT}^{1,2}$ can be written as

$$
\begin{aligned}
E\left[\gamma_{\sigma}\right] & =T\left[\gamma_{\sigma}\right]+E_{\mathrm{ext}}\left[\gamma_{\sigma}\right]+J\left[\gamma_{\sigma}\right]+E_{\mathrm{XC}}\left[\gamma_{\sigma}\right] \\
& =\int \mathrm{d} \mathbf{x} \lim _{\mathbf{x}^{\prime} \rightarrow \mathbf{x}}\left(-\frac{\nabla_{\mathrm{r}}^{2}}{2}\right) \gamma\left[\mathbf{x}, \mathbf{x}^{\prime}\right]+\int \mathrm{d} \mathbf{x} \mathrm{d} \mathbf{x}^{\prime} v_{\mathrm{ext}}\left[\mathbf{x}, \mathbf{x}^{\prime}\right] \gamma\left[\mathbf{x}, \mathbf{x}^{\prime}\right] \\
& +\frac{1}{2} \int \mathrm{d} \mathbf{x} \mathrm{d} \mathbf{x}^{\prime} v_{c}\left[\mathbf{r}, \mathbf{r}^{\prime}\right] \gamma[\mathbf{x}, \mathbf{x}] \gamma\left[\mathbf{x}^{\prime}, \mathbf{x}^{\prime}\right]+E_{\mathrm{XC}}\left[\gamma_{\sigma}\right],
\end{aligned}
$$

where $\gamma_{\sigma}$ denotes the one-electron reduced density matrix (1-RDM), $\mathbf{x} \equiv\{\mathbf{r}, \sigma\}$ is combined space and spin coordinates, $\nabla_{\mathrm{r}}^{2}$ is the Laplace operator, $v_{\mathrm{ext}}\left(\mathbf{x}, \mathbf{x}^{\prime}\right)$ is the external potential,

$v_{c}\left[\mathbf{r}, \mathbf{r}^{\prime}\right]=1 /\left|\mathbf{r}-\mathbf{r}^{\prime}\right|$ and $E_{\mathrm{XC}}\left[\gamma_{\sigma}\right]$ is the exchange-correlation (XC) energy that is obtained by approximation. Given the spectral representation of 1-RDM, i.e. $\gamma_{\sigma}=\sum_{p=1}^{K}\left|\psi_{p}^{\sigma}\right\rangle n_{p}^{\sigma}\left\langle\psi_{p}^{\sigma}\right|$ ( $K$ being the dimension of the basis set), the energy functional is typically minimized with respect to natural orbitals $\left\{\psi_{p}^{\sigma}\right\}$ and natural occupations $\left\{n_{p}^{\sigma}\right\}$ respectively. Unlike occupations in KS-DFT being specified according to the Aufbau principle, natural occupations $\left\{n_{p}^{\sigma}\right\}$ in RDMFT are optimized under the ensemble $N$-representability constraint ${ }^{3,4}$

$$
0 \leq n_{p}^{\sigma} \leq 1
$$

and

$$
h^{\sigma}\left(\left\{n_{p}^{\sigma}\right\}\right)=\sum_{p=1}^{K} n_{p}^{\sigma}-N_{0}^{\sigma}=0,
$$

where $N_{0}^{\sigma}$ is the electron number of $\sigma$ spin.

The commonly used method to optimize the natural occupations is the Lagrange multiplier (LM) method, which has some fundamental limitations such as slow convergence speed and heavy dependence on the initial guess. While the explicit-by-implicit (EBI) method proposed in this work provides a promising alternative to deal with the constrained optimization problem. The power functionals ${ }^{5}$ with different powers $(\omega)$ are used here to demonstrate the effectiveness of EBI in handling the ensemble $N$-representability constraint. The power 
functionals take the form,

$$
E\left[\left\{n_{p}^{\sigma}\right\}\right]=\sum_{\sigma}^{\alpha, \beta} \sum_{p}^{K} H_{p p}^{\sigma} n_{p}^{\sigma}+\frac{1}{2} \sum_{\sigma, \sigma^{\prime}}^{\alpha, \beta} \sum_{p, q}^{K} J_{p q}^{\sigma \sigma^{\prime}} n_{p}^{\sigma} n_{q}^{\sigma^{\prime}}-\frac{1}{2} \sum_{\sigma}^{\alpha, \beta} \sum_{p, q}^{K} K_{p q}^{\sigma}\left(n_{p}^{\sigma} n_{q}^{\sigma}\right)^{\omega}
$$

where $H_{p p}^{\sigma}$ is the diagonal elements of the core-Hamiltonian, $J_{p q}^{\sigma \sigma^{\prime}}$ and $K_{p q}^{\sigma}$ are direct Coulomb and exchange integrals over the natural orbitals respectively.

\section{Occupation Optimization in LM, ALM and EBI}

In this section, the first and second derivatives in LM, ALM and EBI are derived in details, which are needed in the Newton algorithm (Algorithm 1) to optimize the natural occupations throughout this work.

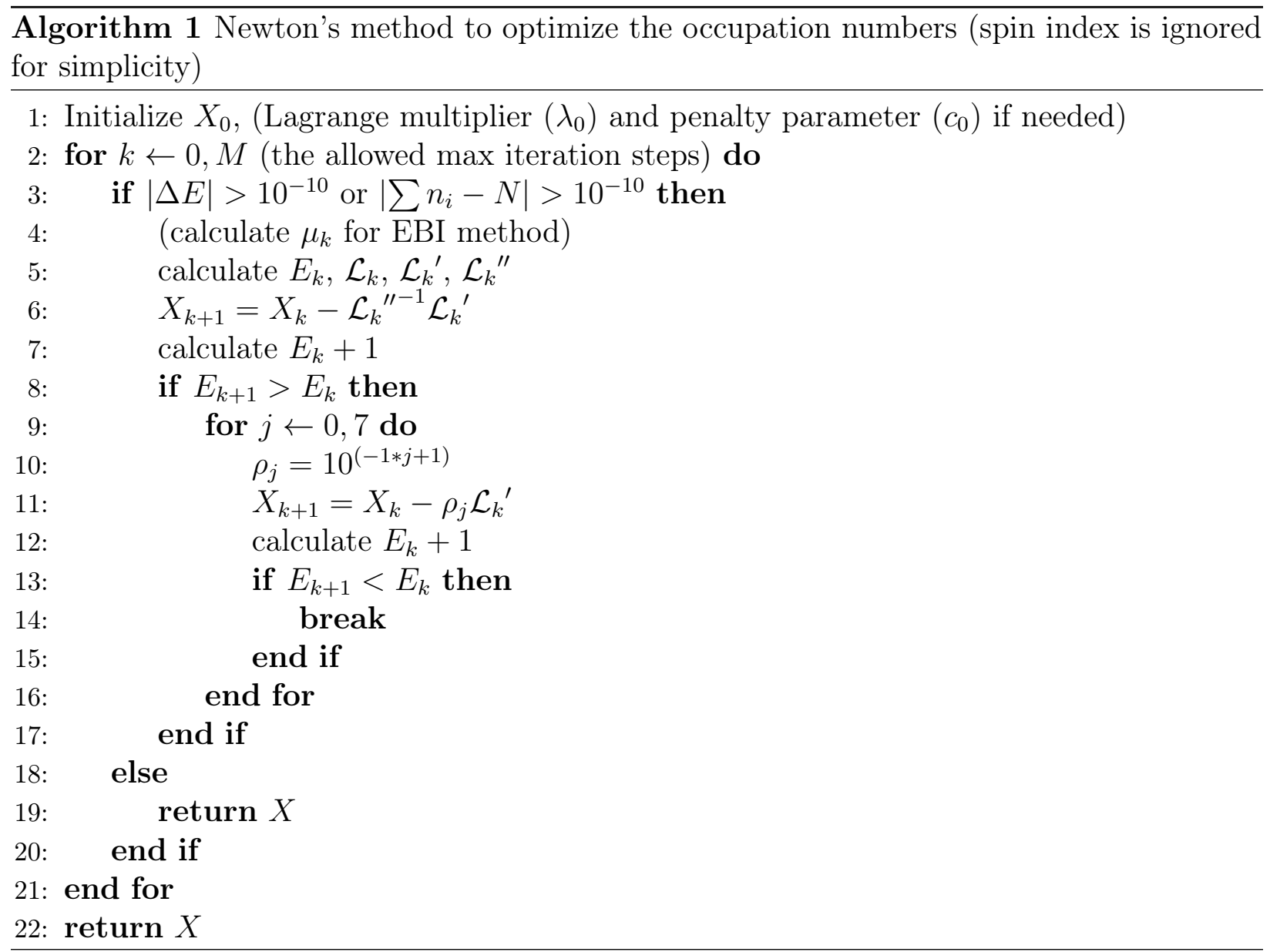




\section{A. The First and Second Derivatives in LM and ALM}

The conventional method to handle the fermionic constraint is by setting

$$
n_{p}^{\sigma}=\cos ^{2} x_{p}^{\sigma},
$$

then the particle number conservation constraint becomes

$$
h^{\sigma}\left(X^{\sigma}\right)=\sum_{p=1}^{K} \cos ^{2} x_{p}^{\sigma}-N_{0}^{\sigma}=0,
$$

where $X^{\sigma}=\left(x_{1}^{\sigma}, x_{2}^{\sigma}, \ldots, x_{K}^{\sigma}\right)^{\mathrm{T}}$.

In $\mathrm{LM},{ }^{6}$ the Lagrangian takes the from

$$
\begin{aligned}
\mathcal{L}\left(X^{\sigma}, \lambda^{\sigma}\right) & =E\left(X^{\sigma}\right)+\sum_{\sigma}^{\alpha, \beta} \lambda^{\sigma} h^{\sigma}\left(X^{\sigma}\right) \\
& =\sum_{\sigma}^{\alpha, \beta} \sum_{p}^{K} H_{p p}^{\sigma} \cos ^{2} x_{p}^{\sigma}+\frac{1}{2} \sum_{\sigma, \sigma^{\prime}}^{\alpha, \beta} \sum_{p, q}^{K} J_{p q}^{\sigma \sigma^{\prime}} \cos ^{2} x_{p}^{\sigma} \cos ^{2} x_{q}^{\sigma^{\prime}} \\
& -\frac{1}{2} \sum_{\sigma}^{\alpha, \beta} \sum_{p, q}^{K} K_{p q}^{\sigma}\left(\cos ^{2} x_{p}^{\sigma} \cos ^{2} x_{q}^{\sigma}\right)^{\omega}+\sum_{\sigma}^{\alpha, \beta} \lambda^{\sigma}\left(\sum_{p} \cos ^{2} x_{p}^{\sigma}-N_{0}^{\sigma}\right),
\end{aligned}
$$

where $\lambda^{\sigma}$ is the Lagrange Multiplier. The formula to update $\left(X^{\sigma}, \lambda^{\sigma}\right)$ in Newton's method is

$$
\left(X^{\sigma}, \lambda^{\sigma}\right)_{(k+1)}=\left(X^{\sigma}, \lambda^{\sigma}\right)_{(k)}-\mathcal{L}^{\prime \prime}\left(X^{\sigma}, \lambda^{\sigma}\right)_{(k)}^{-1} \mathcal{L}^{\prime}\left(X^{\sigma}, \lambda^{\sigma}\right)_{(k)},
$$

where $\mathcal{L}^{\prime}\left(X^{\sigma}, \lambda^{\sigma}\right)$ and $\mathcal{L}^{\prime \prime}\left(X^{\sigma}, \lambda^{\sigma}\right)$ are the first and second derivatives of $\mathcal{L}\left(X^{\sigma}, \lambda^{\sigma}\right)$, which are a vector and a matrix respectively. The first derivatives are

$$
\mathcal{L}^{\prime}\left(X^{\sigma}, \lambda^{\sigma}\right)=\frac{\partial \mathcal{L}\left(X^{\sigma}, \lambda^{\sigma}\right)}{\partial\left(X^{\sigma}, \lambda^{\sigma}\right)}=\left\{\begin{array}{l}
\mathcal{L}_{1}^{\prime}\left(X^{\sigma}, \lambda^{\sigma}\right)=\frac{\partial \mathcal{L}\left(X^{\sigma}, \lambda^{\sigma}\right)}{\partial x_{1}^{\sigma}}, \\
\mathcal{L}_{2}^{\prime}\left(X^{\sigma}, \lambda^{\sigma}\right)=\frac{\partial \mathcal{L}\left(X^{\sigma}, \lambda^{\sigma}\right)}{\partial x_{2}^{\sigma}}, \\
\vdots \\
\mathcal{L}_{K}^{\prime}\left(X^{\sigma}, \lambda^{\sigma}\right)=\frac{\partial \mathcal{L}\left(X^{\sigma}, \lambda^{\sigma}\right)}{\partial x_{K}^{\sigma}}, \\
\mathcal{L}_{K+1}^{\prime}\left(X^{\sigma}, \lambda^{\sigma}\right)=\frac{\partial \mathcal{L}\left(X^{\sigma}, \lambda^{\sigma}\right)}{\partial \lambda^{\sigma}}=\sum_{p}^{K} \cos ^{2} x_{p}^{\sigma}-N_{0}^{\sigma},
\end{array}\right.
$$


where

$$
\begin{aligned}
\mathcal{L}_{p}^{\prime}\left(X^{\sigma}, \lambda^{\sigma}\right) & =\frac{\partial \mathcal{L}\left(X^{\sigma}, \lambda^{\sigma}\right)}{\partial x_{p}^{\sigma}}=\frac{\partial E\left(X^{\sigma}\right)}{\partial x_{p}^{\sigma}}+\lambda^{\sigma} \frac{\partial h^{\sigma}\left(X^{\sigma}\right)}{\partial x_{p}^{\sigma}} \\
& =\frac{\partial E\left(X^{\sigma}\right)}{\partial x_{p}^{\sigma}}-\lambda^{\sigma} \sin 2 x_{p}^{\sigma}, \quad p=1,2, \ldots, K \\
\frac{\partial E\left(X^{\sigma}\right)}{\partial x_{p}^{\sigma}} & =-H_{p p}^{\sigma} \sin 2 x_{p}^{\sigma}-\sum_{\sigma, \sigma^{\prime}}^{\alpha, \beta} \sum_{q}^{K} J_{p q}^{\sigma \sigma^{\prime}} \cos ^{2} x_{q}^{\sigma^{\prime}} \sin 2 x_{p}^{\sigma} \\
& +\omega \sum_{\sigma}^{\alpha, \beta} \sum_{q}^{K} K_{p q}^{\sigma} \cos ^{2 \omega} x_{q}^{\sigma} \cos ^{2 \omega-2} x_{p}^{\sigma} \sin 2 x_{p}^{\sigma} .
\end{aligned}
$$

The second derivatives are

$$
\mathcal{L}^{\prime \prime}\left(X^{\sigma}, \lambda^{\sigma}\right)=\left|\begin{array}{ccccc}
\frac{\partial^{2} \mathcal{L}}{\partial x_{1}^{\sigma 2}} & \frac{\partial^{2} \mathcal{L}}{\partial x_{1}^{\sigma} \partial x_{2}^{\sigma}} & \cdots & \frac{\partial^{2} L}{\partial x_{1}^{\sigma} \partial x_{K}^{\sigma}} & \frac{\partial^{2} \mathcal{L}}{\partial x_{1}^{\sigma} \partial \lambda^{\sigma}} \\
\vdots & \vdots & \vdots & \vdots & \vdots \\
\frac{\partial^{2} \mathcal{L}}{\partial \lambda^{\sigma} \partial x_{1}^{\sigma}} & \frac{\partial^{2} \mathcal{L}}{\partial \lambda^{\sigma} \partial x_{2}^{\sigma}} & \cdots & \frac{\partial^{2} \mathcal{L}}{\partial \lambda^{\sigma} \partial x_{K}^{\sigma}} & \frac{\partial^{2} \mathcal{L}}{\partial \lambda^{\sigma 2}}
\end{array}\right|
$$

where

$$
\begin{gathered}
\mathcal{L}_{p p}^{\prime \prime}\left(X^{\sigma}, \lambda^{\sigma}\right)=\frac{\partial^{2} \mathcal{L}\left(X^{\sigma}, \lambda^{\sigma}\right)}{\partial x_{p}^{\sigma 2}}=\frac{\partial^{2} E\left(X^{\sigma}\right)}{\partial x_{p}^{\sigma 2}}+\lambda^{\sigma} \frac{\partial^{2} h^{\sigma}\left(X^{\sigma}\right)}{\partial x_{p}^{\sigma 2}} \\
=\frac{\partial^{2} E\left(X^{\sigma}\right)}{\partial x_{p}^{\sigma 2}}-2 \lambda^{\sigma} \cos 2 x_{p}^{\sigma}, \quad p=1,2, \ldots K \\
\frac{\partial^{2} E\left(X^{\sigma}\right)}{\partial x_{p}^{\sigma 2}}=-2 H_{p}^{\sigma} \cos 2 x_{p}^{\sigma}-2 \sum_{\sigma, \sigma^{\prime}}^{\alpha, \beta} \sum_{q}^{K} J_{p q}^{\sigma \sigma^{\prime}} \cos ^{2} x_{q}^{\sigma^{\prime}} \cos 2 x_{p}^{\sigma} \\
+\omega \sum_{q}^{K} \sum_{\sigma}^{\alpha, \beta} K_{p q}^{\sigma} \cos ^{2 \omega} x_{q}^{\sigma}\left[2 \cos 2 x_{p}^{\sigma} \cos ^{2 \omega-2} x_{p}^{\sigma}-(\omega-1) \sin ^{2} 2 x_{p}^{\sigma} \cos ^{2 \omega-4} x_{p}^{\sigma}\right] \\
+J_{p p}^{\sigma \sigma} \sin ^{2} 2 x_{p}^{\sigma}-\omega^{2} K_{p p}^{\sigma} \cos ^{4 \omega-4} x_{p}^{\sigma} \sin ^{2} 2 x_{p}^{\sigma}, \\
\mathcal{L}_{p q}^{\prime \prime}\left(X^{\sigma}, \lambda^{\sigma}\right)=\frac{\partial^{2} \mathcal{L}\left(X^{\sigma}, \lambda^{\sigma}\right)}{\partial x_{p}^{\sigma} \partial x_{q}^{\sigma}}=\frac{\partial^{2} E\left(X^{\sigma}\right)}{\partial x_{p}^{\sigma} \partial x_{q}^{\sigma}}+\lambda^{\sigma} \frac{\partial^{2} h^{\sigma}\left(X^{\sigma}\right)}{\partial x_{p}^{\sigma} \partial x_{q}^{\sigma}} \\
=\frac{\partial^{2} E\left(X^{\sigma}\right)}{\partial x_{p}^{\sigma} \partial x_{q}^{\sigma}}, p \neq q, 1 \leq p_{p} \leq K \\
\frac{\partial^{2} E\left(X^{\sigma}\right)}{\partial x_{p}^{\sigma} \partial x_{q}^{\sigma}}=J_{p q}^{\sigma \sigma^{\prime}} \sin 2 x_{p}^{\sigma} \sin 2 x_{q}^{\sigma^{\prime}}-\omega^{2} K_{p q}^{\sigma} \cos ^{2 \omega-2} x_{q}^{\sigma} \cos ^{2 \omega-2} x_{p}^{\sigma} \sin 2 x_{p}^{\sigma} \sin 2 x_{q}^{\sigma}
\end{gathered}
$$




$$
\begin{gathered}
\mathcal{L}_{p, K+1}^{\prime \prime}\left(X^{\sigma}, \lambda^{\sigma}\right)=\mathcal{L}_{K+1, p}^{\prime \prime}\left(X^{\sigma}, \lambda^{\sigma}\right)=\frac{\partial^{2} \mathcal{L}}{\partial x_{p}^{\sigma} \partial \lambda^{\sigma}}=-\sin 2 x_{p}^{\sigma}, \\
\mathcal{L}_{K+1, K+1}^{\prime \prime}\left(X^{\sigma}, \lambda^{\sigma}\right)=\frac{\partial^{2} \mathcal{L}}{\partial \lambda^{\sigma 2}}=0 .
\end{gathered}
$$

The augmented LM (ALM) method is a common way to improve the LM method, by adding a penalty to the Lagrangian. ${ }^{6}$ The augmented Lagrangian considered in this work takes the form

$$
\mathcal{L}_{a}\left(X^{\sigma}\right)=E\left(X^{\sigma}\right)+\sum_{\sigma}^{\alpha, \beta} \lambda_{k}^{\sigma}\left(\sum_{p}^{K} \cos ^{2} x_{p}^{\sigma}-N_{0}^{\sigma}\right)+\sum_{\sigma}^{\alpha, \beta} \frac{c_{k}^{\sigma}}{2}\left(\sum_{p}^{K} \cos ^{2} x_{p}^{\sigma}-N_{0}^{\sigma}\right)^{2} .
$$

In ALM, $X^{\sigma}$ are optimized at a series of $c_{k}^{\sigma}$ and $\lambda_{k}^{\sigma}$ that are updated by $c_{k}^{\sigma}=2^{k}$ and $\lambda_{k}^{\sigma}=\lambda_{k-1}^{\sigma}+c_{k-1}^{\sigma} h^{\sigma}\left(X_{k-1}^{\sigma}\right)$. As $c_{k}^{\sigma}$ and $\lambda_{k}^{\sigma}$ are updated, the $X_{k}^{\sigma}$ optimized at each fixed $c_{k}^{\sigma}$ and $\lambda_{k}^{\sigma}$ will approach the optimal solution progressively. The first derivatives of $\mathcal{L}_{a}\left(X^{\sigma}\right)$ are

$$
\mathcal{L}_{a}^{\prime}\left(X^{\sigma}\right)=\frac{\partial \mathcal{L}_{a}\left(X^{\sigma}\right)}{\partial x_{p}^{\sigma}}=\frac{\partial E\left(X^{\sigma}\right)}{\partial x_{p}^{\sigma}}-\lambda_{k}^{\sigma} \sin 2 x_{p}^{\sigma}-c_{k}^{\sigma} \sin 2 x_{p}^{\sigma}\left(\sum_{q}^{K} \cos ^{2} x_{q}^{\sigma}-N_{0}^{\sigma}\right),
$$

and the second derivatives of $\mathcal{L}_{a}\left(X^{\sigma}\right)$ are

$$
\begin{aligned}
\mathcal{L}_{a}^{\prime \prime}\left(X^{\sigma}\right)_{p p} & =\frac{\partial^{2} \mathcal{L}_{a}\left(X^{\sigma}\right)}{\partial x_{p}^{\sigma 2}} \\
& =\frac{\partial^{2} E\left(X^{\sigma}\right)}{\partial x_{p}^{\sigma 2}}-2 \lambda_{k}^{\sigma} \cos 2 x_{p}^{\sigma}-2 c_{k}^{\sigma} \cos 2 x_{p}^{\sigma}\left(\sum_{q}^{K} \cos ^{2} x_{q}^{\sigma}-N_{0}^{\sigma}\right)+c_{k}^{\sigma} \sin ^{2} 2 x_{p}^{\sigma},
\end{aligned}
$$

and

$$
\mathcal{L}_{a}^{\prime \prime}\left(X^{\sigma}\right)_{p q}=\frac{\partial^{2} \mathcal{L}_{a}\left(X^{\sigma}\right)}{\partial x_{p}^{\sigma} \partial x_{q}^{\sigma}}=\frac{\partial^{2} E\left(X^{\sigma}\right)}{\partial x_{p}^{\sigma} \partial x_{q}^{\sigma}}+c_{k}^{\sigma} \sin 2 x_{p}^{\sigma} \sin 2 x_{q}^{\sigma}, p \neq q
$$

\section{B. The First and Second Derivatives in EBI}

To address the convergence issues of LM and ALM, the idea of EBI for handling the ensemble $N$-representability constraint is introduced in this work. The natural occupations in EBI are defined as $n_{p}^{\sigma}=n\left(x_{p}^{\sigma}, \mu^{\sigma}\right)$, which depend not only on the dedicated variable $x_{p}^{\sigma}$ corresponding to each occupation but also on the common variable $\mu^{\sigma}$ to all occupations. $\mu^{\sigma}$ is actually 
an implicit function of $X^{\sigma}$, which is brought in to consider the nonlinear unclear connection among $X^{\sigma}$, embedded in

$$
N\left(X^{\sigma}, \mu^{\sigma}\right)=\sum_{p=1}^{K} n\left(x_{p}^{\sigma}, \mu^{\sigma}\right)=N_{0}^{\sigma} .
$$

The error function is used to represent the occupations in this work,

$$
n_{p}^{\sigma}=n\left(x_{p}^{\sigma}, \mu^{\sigma}\right)=\frac{\operatorname{erf}\left(x_{p}^{\sigma}+\mu^{\sigma}\right)+1}{2} .
$$

As the error function is a monotonic function (see Fig. S1), for any set of $\left\{x_{p}^{\sigma}\right\}$, there is a unique value for $\mu^{\sigma}$ that satisfies Eq. S23. Hence, the solution of $\mu^{\sigma}$ is just a one-dimension optimization problem, which can be easily solved with the Newton's algorithm (see Algorithm 2). In fact, other sigmoid functions are also applicable here besides the error function.

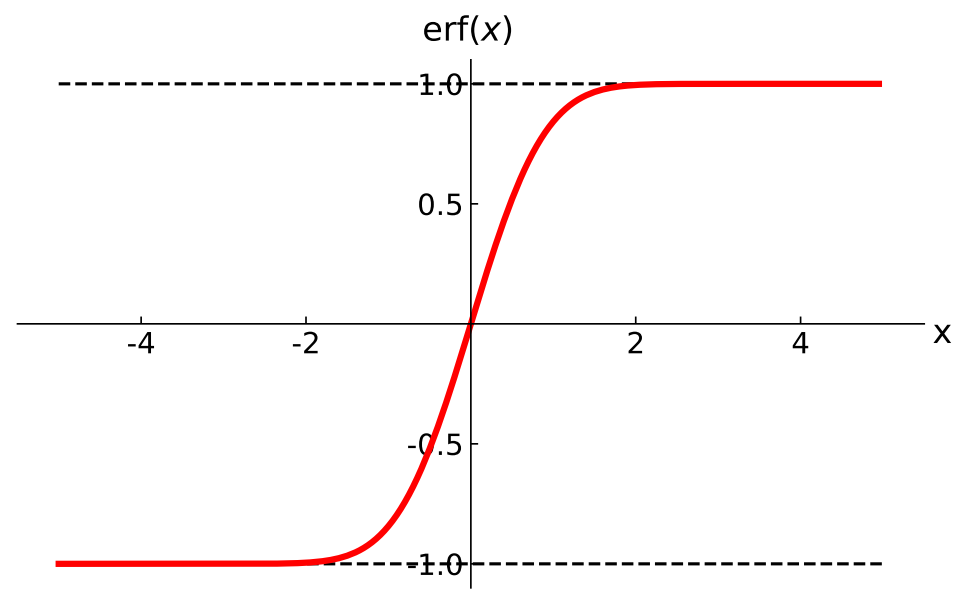

Figure S1: Plot of the error function.

In fact, only the first and second derivatives of $\mu^{\sigma}$ are needed during optimization, which are derived below. For the first derivatives,

$$
\begin{gathered}
\sum_{p}^{K} \mathrm{~d} n_{p}^{\sigma}=\frac{1}{2} \sum_{p}^{K} \operatorname{erf}^{\prime}\left(x_{p}^{\sigma}+\mu^{\sigma}\right)\left(\mathrm{d} x_{p}^{\sigma}+\mathrm{d} \mu^{\sigma}\right)=0 \\
\Rightarrow \mathrm{d} \mu^{\sigma}=-\frac{\sum_{p}^{K} \operatorname{erf}^{\prime}\left(x_{p}^{\sigma}+\mu^{\sigma}\right) \mathrm{d} x_{p}^{\sigma}}{\sum_{p}^{K} \operatorname{erf}^{\prime}\left(x_{p}^{\sigma}+\mu^{\sigma}\right)}
\end{gathered}
$$




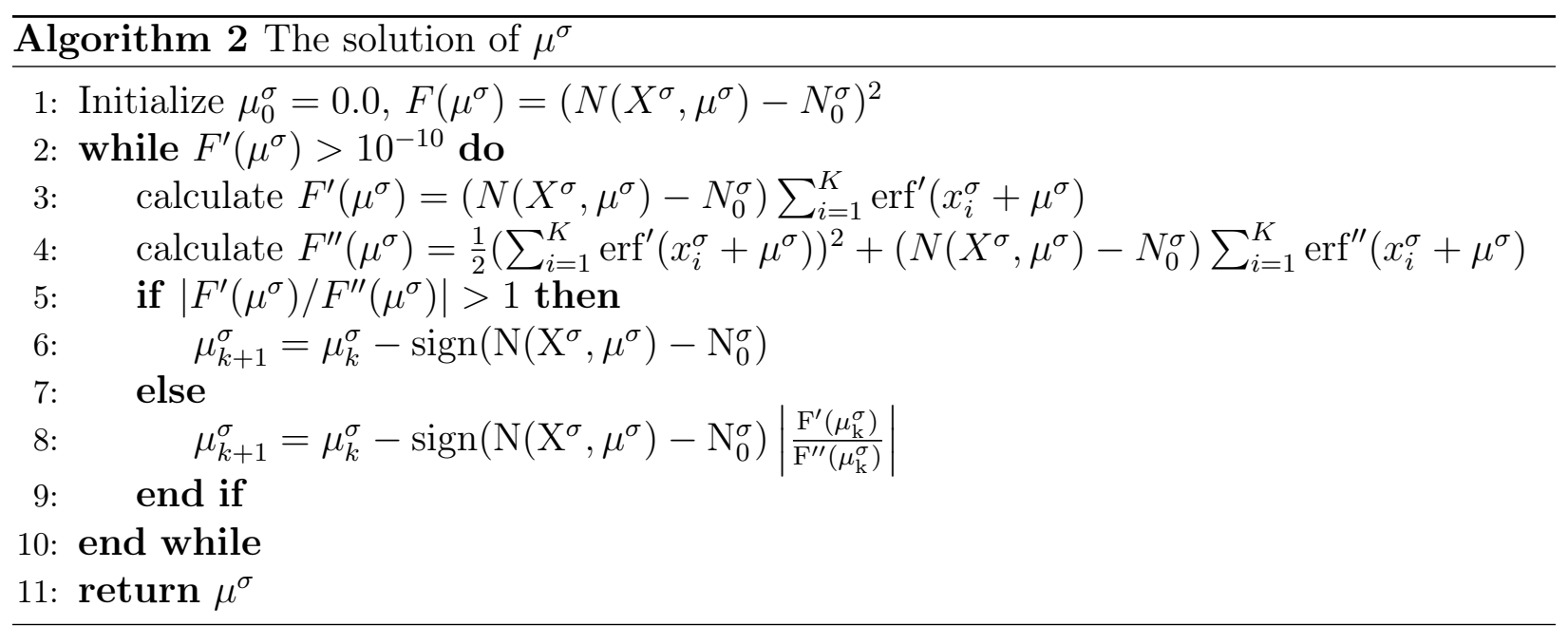

hence

$$
\frac{\partial \mu^{\sigma}}{\partial x_{p}^{\sigma}}=-\frac{\operatorname{erf}^{\prime}\left(x_{p}^{\sigma}+\mu^{\sigma}\right)}{\sum_{q}^{K} \operatorname{erf}^{\prime}\left(x_{q}^{\sigma}+\mu^{\sigma}\right)} .
$$

For the second derivatives,

$$
\begin{aligned}
\sum_{p}^{K} \mathrm{~d}^{2} n_{p}^{\sigma} & =\frac{1}{2} \sum_{p}^{K} \operatorname{erf}^{\prime \prime}\left(x_{p}^{\sigma}+\mu^{\sigma}\right)\left(\mathrm{d} x_{p}^{\sigma}+\mathrm{d} \mu^{\sigma}\right)^{2}+\frac{1}{2} \sum_{p}^{K} \operatorname{erf}^{\prime}\left(x_{p}^{\sigma}+\mu^{\sigma}\right) \mathrm{d}^{2} \mu^{\sigma}=0 \\
\Rightarrow \mathrm{d}^{2} \mu^{\sigma} & =-\frac{\sum_{p}^{K} \operatorname{erf}^{\prime \prime}\left(x_{p}^{\sigma}+\mu^{\sigma}\right)\left(\mathrm{d} x_{p}^{\sigma}+\mathrm{d} \mu^{\sigma}\right)^{2}}{\sum_{p}^{K} \operatorname{erf}^{\prime}\left(x_{p}^{\sigma}+\mu^{\sigma}\right)} \\
& =-\frac{\sum_{p}^{K} \operatorname{erf}^{\prime \prime}\left(x_{p}^{\sigma}+\mu^{\sigma}\right)\left(\mathrm{d} x_{p}^{\sigma} \mathrm{d} x_{p}^{\sigma}+\mathrm{d} \mu^{\sigma} \mathrm{d} x_{p}^{\sigma}+\mathrm{d} x_{p}^{\sigma} \mathrm{d} \mu^{\sigma}+\mathrm{d} \mu^{\sigma} \mathrm{d} \mu^{\sigma}\right)}{\sum_{p}^{K} \operatorname{erf}^{\prime}\left(x_{p}^{\sigma}+\mu^{\sigma}\right)}
\end{aligned}
$$

hence

$$
\frac{\partial^{2} \mu^{\sigma}}{\partial x_{p}^{\sigma 2}}=-\frac{\operatorname{erf}^{\prime \prime}\left(x_{p}^{\sigma}+\mu^{\sigma}\right)+2 \operatorname{erf}^{\prime \prime}\left(x_{p}^{\sigma}+\mu^{\sigma}\right) \frac{\partial \mu^{\sigma}}{\partial x_{p}^{\sigma}}+\sum_{q}^{K} \operatorname{erf}^{\prime \prime}\left(x_{q}^{\sigma}+\mu^{\sigma}\right) \frac{\partial \mu^{\sigma}}{\partial x_{q}^{\sigma}} \frac{\partial \mu^{\sigma}}{\partial x_{q}^{\sigma}}}{\sum_{q}^{K} \operatorname{erf}^{\prime}\left(x_{q}^{\sigma}+\mu^{\sigma}\right)}
$$




$$
\frac{\partial^{2} \mu^{\sigma}}{\partial x_{p}^{\sigma} \partial x_{q}^{\sigma}}=-\frac{\operatorname{erf}^{\prime \prime}\left(x_{q}^{\sigma}+\mu^{\sigma}\right) \frac{\partial \mu^{\sigma}}{\partial x_{p}^{\sigma}}+\operatorname{erf}^{\prime \prime}\left(x_{p}^{\sigma}+\mu^{\sigma}\right) \frac{\partial \mu^{\sigma}}{\partial x_{q}^{\sigma}}+\sum_{k}^{K} \operatorname{erf}^{\prime \prime}\left(x_{k}^{\sigma}+\mu^{\sigma}\right) \frac{\partial \mu^{\sigma}}{\partial x_{p}^{\sigma}} \frac{\partial \mu^{\sigma}}{\partial x_{q}^{\sigma}}}{\sum_{k}^{K} \operatorname{erf}^{\prime}\left(x_{k}^{\sigma}+\mu^{\sigma}\right)} .
$$

Here, the first and second derivatives of the error function are

$$
\begin{gathered}
\operatorname{erf}^{\prime}(x)=\frac{2}{\sqrt{\pi}} e^{-x^{2}}, \\
\operatorname{erf}^{\prime \prime}(x)=-\frac{4}{\sqrt{\pi}} x e^{-x^{2}} .
\end{gathered}
$$

In $\mathrm{EBI}$, the objective function is the energy functional,

$$
E\left(X^{\sigma}\right)=E\left(\left\{\operatorname{erf}\left(x_{p}^{\sigma}+\mu^{\sigma}\right)\right\}\right) .
$$

Making use of Eqs. S26, S28 and S29, the first and second derivatives of the energy functional can be calculated. The first derivatives of $E\left(X^{\sigma}\right)$ are

$$
\begin{aligned}
E^{\prime}\left(X^{\sigma}\right)_{p} & =\frac{\partial E\left(X^{\sigma}\right)}{\partial x_{p}^{\sigma}}=\sum_{\sigma}^{\alpha, \beta} \sum_{q}^{K} H_{q q}^{\sigma} \frac{\partial n_{q}^{\sigma}}{\partial x_{p}^{\sigma}}+\frac{1}{2} \sum_{\sigma, \sigma^{\prime}}^{\alpha, \beta} \sum_{k, l}^{K} J_{k l}^{\sigma \sigma^{\prime}}\left(\frac{\partial n_{k}^{\sigma}}{\partial x_{p}^{\sigma}} n_{l}^{\sigma^{\prime}}+\frac{\partial n_{l}^{\sigma^{\prime}}}{\partial x_{p}^{\sigma}} n_{k}^{\sigma}\right) \\
& -\frac{\omega}{2} \sum_{\sigma}^{\alpha, \beta} \sum_{k, l}^{K} K_{k l}^{\sigma}\left(n_{k}^{\sigma} n_{l}^{\sigma}\right)^{\omega-1}\left(n_{k}^{\sigma} \frac{\partial n_{l}^{\sigma}}{\partial x_{p}^{\sigma}}+\frac{\partial n_{k}^{\sigma}}{\partial x_{p}^{\sigma}} n_{l}^{\sigma}\right),
\end{aligned}
$$

where

$$
\frac{\partial n_{p}^{\sigma}}{\partial x_{p}^{\sigma}}=\frac{1}{2} \operatorname{erf}^{\prime}\left(x_{p}^{\sigma}+\mu^{\sigma}\right)\left(1+\frac{\partial \mu^{\sigma}}{\partial x_{p}^{\sigma}}\right),
$$

and

$$
\frac{\partial n_{q}^{\sigma}}{\partial x_{p}^{\sigma}}=\frac{1}{2} \operatorname{erf}^{\prime}\left(x_{q}^{\sigma}+\mu^{\sigma}\right) \frac{\partial \mu^{\sigma}}{\partial x_{p}^{\sigma}}, p \neq q .
$$

The second derivatives of $E\left(X^{\sigma}\right)$ are

$$
\begin{aligned}
E^{\prime \prime}\left(X^{\sigma}\right)_{p p} & =\frac{\partial^{2} E\left(X^{\sigma}\right)}{\partial x_{p}^{\sigma 2}}=\sum_{\sigma}^{\alpha, \beta} \sum_{k}^{K} H_{k k}^{\sigma} \frac{\partial^{2} n_{k}^{\sigma}}{\partial x_{p}^{\sigma 2}}+\sum_{\sigma, \sigma^{\prime}}^{\alpha, \beta} \sum_{k, l}^{K} J_{k l}^{\sigma \sigma^{\prime}}\left(\frac{\partial^{2} n_{k}^{\sigma}}{\partial x_{p}^{\sigma 2}} n_{l}^{\sigma^{\prime}}+\frac{\partial n_{l}^{\sigma^{\prime}}}{\partial x_{p}^{\sigma}} \frac{\partial n_{k}^{\sigma}}{\partial x_{p}^{\sigma}}\right) \\
& -\omega \sum_{\sigma}^{\alpha, \beta} \sum_{k, l}^{K} K_{k l}^{\sigma}\left(n_{k}^{\sigma} n_{l}^{\sigma}\right)^{\omega-1}\left(\frac{\partial^{2} n_{k}^{\sigma}}{\partial x_{p}^{\sigma 2}} n_{l}^{\sigma}+\frac{\partial n_{l}^{\sigma}}{\partial x_{p}^{\sigma}} \frac{\partial n_{k}^{\sigma}}{\partial x_{p}^{\sigma}}\right) \\
& -\frac{\omega(\omega-1)}{2}\left(n_{k}^{\sigma} n_{l}^{\sigma}\right)^{\omega-2} \sum_{\sigma}^{\alpha, \beta} \sum_{k, l}^{K} K_{k l}^{\sigma}\left(n_{k}^{\sigma} \frac{\partial n_{l}^{\sigma}}{\partial x_{p}^{\sigma}}+\frac{\partial n_{k}^{\sigma}}{\partial x_{p}^{\sigma}} n_{l}^{\sigma}\right)^{2},
\end{aligned}
$$


and

$$
\begin{aligned}
E^{\prime \prime}\left(X^{\sigma}\right)_{p q} & =\frac{\partial^{2} E\left(X^{\sigma}\right)}{\partial x_{p}^{\sigma} \partial x_{q}^{\sigma}}=\sum_{\sigma}^{\alpha, \beta} \sum_{k}^{K} H_{k k}^{\sigma} \frac{\partial^{2} n_{k}^{\sigma}}{\partial x_{p}^{\sigma} \partial x_{q}^{\sigma}}+\sum_{\sigma, \sigma^{\prime}}^{\alpha, \beta} \sum_{k, l}^{K} J_{k l}^{\sigma \sigma^{\prime}}\left(\frac{\partial^{2} n_{k}^{\sigma}}{\partial x_{p}^{\sigma} \partial x_{q}^{\sigma}} n_{l}^{\sigma^{\prime}}+\frac{\partial n_{l}^{\sigma^{\prime}}}{\partial x_{p}^{\sigma}} \frac{\partial n_{k}^{\sigma}}{\partial x_{q}^{\sigma}}\right) \\
& -\omega \sum_{\sigma}^{\alpha, \beta} \sum_{k, l}^{K} K_{k l}^{\sigma}\left(n_{k}^{\sigma} n_{l}^{\sigma}\right)^{\omega-1}\left(\frac{\partial^{2} n_{l}^{\sigma}}{\partial x_{p}^{\sigma} \partial x_{q}^{\sigma}} n_{k}^{\sigma}+\frac{\partial n_{k}^{\sigma}}{\partial x_{p}^{\sigma}} \frac{\partial n_{l}^{\sigma}}{\partial x_{q}^{\sigma}}\right) \\
& -\frac{\omega(\omega-1)}{2}\left(n_{k}^{\sigma} n_{l}^{\sigma}\right)^{\omega-2} \sum_{\sigma}^{\alpha, \beta} \sum_{k, l}^{K} K_{k l}^{\sigma}\left(n_{k}^{\sigma} \frac{\partial n_{l}^{\sigma}}{\partial x_{p}^{\sigma}}+\frac{\partial n_{k}^{\sigma}}{\partial x_{p}^{\sigma}} n_{l}^{\sigma}\right)\left(n_{k}^{\sigma} \frac{\partial n_{l}^{\sigma}}{\partial x_{q}^{\sigma}}+\frac{\partial n_{k}^{\sigma}}{\partial x_{q}^{\sigma}} n_{l}^{\sigma}\right),
\end{aligned}
$$

where

$$
\begin{gathered}
\frac{\partial^{2} n_{p}^{\sigma}}{\partial x_{p}^{\sigma 2}}=\frac{1}{2} \operatorname{erf}^{\prime \prime}\left(x_{p}^{\sigma}+\mu^{\sigma}\right)\left(1+\frac{\partial \mu^{\sigma}}{\partial x_{p}^{\sigma}}\right)^{2}+\frac{1}{2} \operatorname{erf}^{\prime}\left(x_{p}^{\sigma}+\mu^{\sigma}\right) \frac{\partial^{2} \mu^{\sigma}}{\partial x_{p}^{2}} \\
\frac{\partial^{2} n_{q}^{\sigma}}{\partial x_{p}^{\sigma 2}}=\frac{1}{2} \operatorname{erf}^{\prime \prime}\left(x_{q}^{\sigma}+\mu^{\sigma}\right) \frac{\partial \mu^{\sigma}}{\partial x_{p}^{\sigma}} \frac{\partial \mu^{\sigma}}{\partial x_{p}^{\sigma}}+\frac{1}{2} \operatorname{erf}^{\prime}\left(x_{q}^{\sigma}+\mu^{\sigma}\right) \frac{\partial^{2} \mu^{\sigma}}{\partial x_{p}^{\sigma 2}} \\
\frac{\partial^{2} n_{p}^{\sigma}}{\partial x_{p}^{\sigma} \partial x_{q}^{\sigma}}=\frac{1}{2} \operatorname{erf}^{\prime \prime}\left(x_{p}^{\sigma}+\mu^{\sigma}\right) \frac{\partial \mu^{\sigma}}{\partial x_{q}^{\sigma}}\left(1+\frac{\partial \mu^{\sigma}}{\partial x_{p}^{\sigma}}\right)+\frac{1}{2} \operatorname{erf}^{\prime}\left(x_{p}^{\sigma}+\mu^{\sigma}\right) \frac{\partial^{2} \mu^{\sigma}}{\partial x_{p}^{\sigma} \partial x_{q}^{\sigma}} \\
\frac{\partial^{2} n_{k}^{\sigma}}{\partial x_{p}^{\sigma} \partial x_{q}^{\sigma}}=\frac{1}{2} \operatorname{erf}^{\prime \prime}\left(x_{k}^{\sigma}+\mu^{\sigma}\right) \frac{\partial \mu^{\sigma}}{\partial x_{p}^{\sigma}} \frac{\partial \mu^{\sigma}}{\partial x_{q}^{\sigma}}+\frac{1}{2} \operatorname{erf}^{\prime}\left(x_{k}^{\sigma}+\mu^{\sigma}\right) \frac{\partial^{2} \mu^{\sigma}}{\partial x_{p}^{\sigma} \partial x_{q}^{\sigma}}, \quad k \neq p, q .
\end{gathered}
$$

\section{Computational Details}

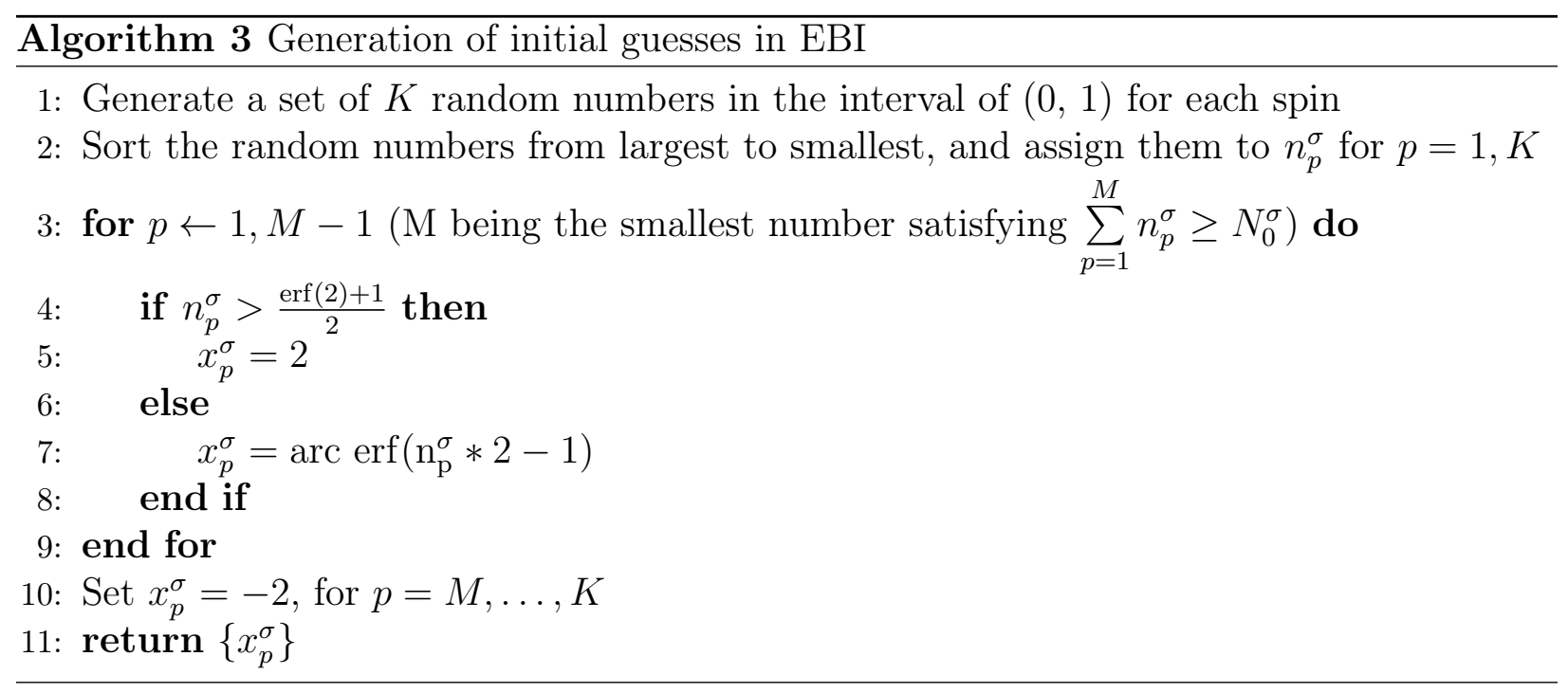

All calculations were performed using a local modified version of the $\mathrm{NWChem}^{7}$ package. 
The power functionals, ${ }^{5}$ i.e. $E_{\mathrm{XC}}\left[\gamma_{\sigma}\right]=-\frac{1}{2} \sum_{\sigma}^{\alpha, \beta} \iint\left|\gamma_{\sigma}^{\omega}\left(\mathbf{r}, \mathbf{r}^{\prime}\right)\right|^{2}\left|\mathbf{r}-\mathbf{r}^{\prime}\right|^{-1} \mathrm{~d}^{3} \mathbf{r d}^{3} \mathbf{r}^{\prime}$ with $\omega$ ranging from 0.1 to 1.0, are used to check the applicability of EBI to different 1-RDM functionals. Unless otherwise specified, the basis set cc-pVTZ ${ }^{8,9}$ is used for all calculations. The input orbitals are optimized by Hartree-Fock(HF) method. The convergence criterion for both energy and occupations is 1e-10. In order to effectively optimize the occupations, here set up a method to generate reasonable initial guesses with random numbers. Algorithm 3 describes the method to generate the initial guesses of $\left\{x_{p}^{\sigma}\right\}$ and occupations in EBI. To obtain the same initial occupations for LM, ALM and EBI, the initial $\left\{x_{p}^{\sigma}\right\}$ in both LM and ALM are obtained via $\arccos \left(\sqrt{n_{p}^{\sigma}}\right)$ and inserting the initial occupations of EBI. The negative of the energy of highest occupied molecular orbital(HOMO) is used as the initial guess of $\lambda^{\sigma}$ in LM. The initial values of $\lambda^{\sigma}$ and $c^{\sigma}$ in ALM are set to 0.0 and 1.0 respectively.

\section{Supplemental Results}

Here provide more test results. In order to make a clear comparison between LM, ALM and EBI, $\omega=0.1$ was taken in Fig. 1 of the main text. In fact, the same results can be obtained for other $\omega$, Fig. S2 shows the results at $\omega=0.5$ as a supplement to Fig. 1 of the main text.

Fig. 2a-c of the main text show the changes of energy, electron number, and gradient of $\mathrm{C}_{6} \mathrm{H}_{6}$ during the natural occupation optimization with the same initial guess. As can be seen, the EBI method not only converges faster than LM and ALM method, but also has lower convergence energy. LM and ALM normally have much worse performance as compared to EBI, especially, LM does not converge with most of the initial guesses in Fig. 2d of the main text. Therefore, the data in Fig. 2a-c of the main text are obtained by trying 10 different initial guesses for LM, and taking the best one as the initial guess for both ALM and EBI as well. Fig. S3 and Fig. S4 provide two examples where the occupation optimization does not converge in 5000 iterations and shows serious numerical problem. In comparison, ALM shows less dependence on the initial guess due to the introduction of the penalty, but there are still cases where the iterations do not converge in 5000 iterations, see Fig. S5. 
Fig. 3a of the main text shows the performance of LM, ALM and EBI on $\mathrm{N}_{2}$ with increasing bond lengths. For both LM and ALM, the optimization was carried out 10 times with different initial guesses, only the lowest energies are recorded in the figure. Fig. S6 shows the test results of the three methods by using the same initial guesses, as can be seen, LM does not converge for most cases. Note that there are still some points that converge to higher energies for the data in Fig. 3b, which are solved by introducing convergence results of adjacent points as initial guesses.

For a complete comparison, here we also check the performance of LM and ALM with the fermionic constraint considered via the error function, i.e. Eq. S24 without the implicit function $\mu^{\sigma}$, instead of the cosine function Eq. S5 that is used throughout the main text. Figs. S7-S11 show that the performances of LM and ALM with the error function, which are actually worse than LM and ALM with the cosine function. In Fig. S11, 50 random initial guesses are used to check the performance of LM with the error function, but none of them converge.

In order to examine the impact of different choices of sigmoid functions for natural occupations on EBI, three sigmoid functions are checked, which invoke error function (Eq. S24), hyperbolic tangent function,

$$
n_{p}^{\sigma}=n\left(x_{p}^{\sigma}, \mu^{\sigma}\right)=\frac{1}{2}\left(\frac{e^{\left(x_{p}^{\sigma}+\mu^{\sigma}\right)}-e^{-\left(x_{p}^{\sigma}+\mu^{\sigma}\right)}}{e^{\left(x_{p}^{\sigma}+\mu^{\sigma}\right)}+e^{-\left(x_{p}^{\sigma}+\mu^{\sigma}\right)}}+1\right),
$$

and modified hyperbolic tangent function,

$$
n_{p}^{\sigma}=n\left(x_{p}^{\sigma}, \mu^{\sigma}\right)=\frac{1}{2}\left(\frac{e^{2\left(x_{p}^{\sigma}+\mu^{\sigma}\right)}-e^{-2\left(x_{p}^{\sigma}+\mu^{\sigma}\right)}}{e^{2\left(x_{p}^{\sigma}+\mu^{\sigma}\right)}+e^{-2\left(x_{p}^{\sigma}+\mu^{\sigma}\right)}}+1\right) .
$$

As shown in Fig. S12, the EBI method with different sigmoid functions show much better performance than the LM methods. Different choices of sigmoid functions for natural occupations rarely affect the results of the natural occupation optimization of $\mathrm{C}_{6} \mathrm{H}_{6}$, only the converged rates are slightly different. 

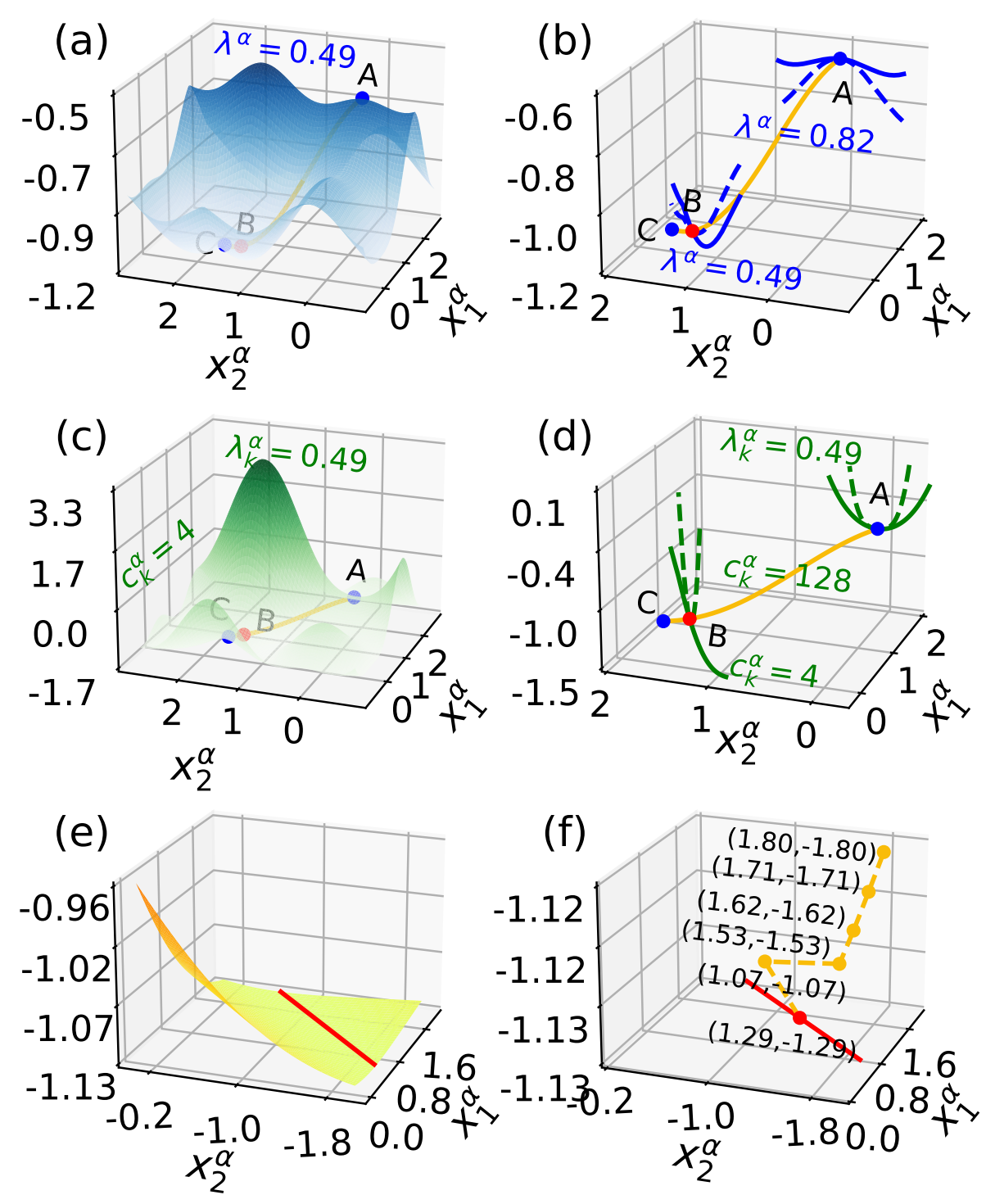

Figure S2: Objective functions of LM, ALM, and EBI for $\mathrm{H}_{2}^{+}$with the minimal basis set. They are (a) the Lagrangian of Eq. S7 with $\lambda^{\alpha}=0.49$; (b) the Lagrangian of Eq. S7 with different $\lambda^{\alpha}$ : $\lambda^{\alpha}=0.49$ (solid curves) and $\lambda^{\alpha}=0.82$ (dashed curves); (c) the augmented Lagrangian of Eq. S19 with $\lambda_{k}^{\alpha}=0.49$ and $c_{k}^{\alpha}=4$; (d) the augmented Lagrangian of Eq. S19 with $\lambda_{k}^{\alpha}=0.49$ and different $c_{k}^{\alpha}: c_{k}^{\alpha}=4$ (solid curves) and $c_{k}^{\alpha}=128$ (dashed curves); (e) the electronic energy as an implicit function of $\left(x_{1}^{\alpha}, x_{2}^{\alpha}\right)$ through Eq. S24, (f) the energy optimized with respect to $\left(x_{1}^{\alpha}, x_{2}^{\alpha}\right)$ in EBI. A, B, and $\mathrm{C}$ in $(\mathrm{a})-(\mathrm{d})$ are critical points. The points that satisfy the particle number conservation constraint (i.e. Eq. S3) are in yellow, and the optimal solutions are in red. Hence, the points that satisfy Eq. S3 form a curve in LM and ALM, and the optimal solution is a point (i.e. $B$ ) in the curve; In contrast, all points satisfy Eq. S3 in EBI, and the optimal solutions are a line (all points in the line giving the same occupations and energy). The power functional with $\omega=0.5$ is applied. All the data are in au. 


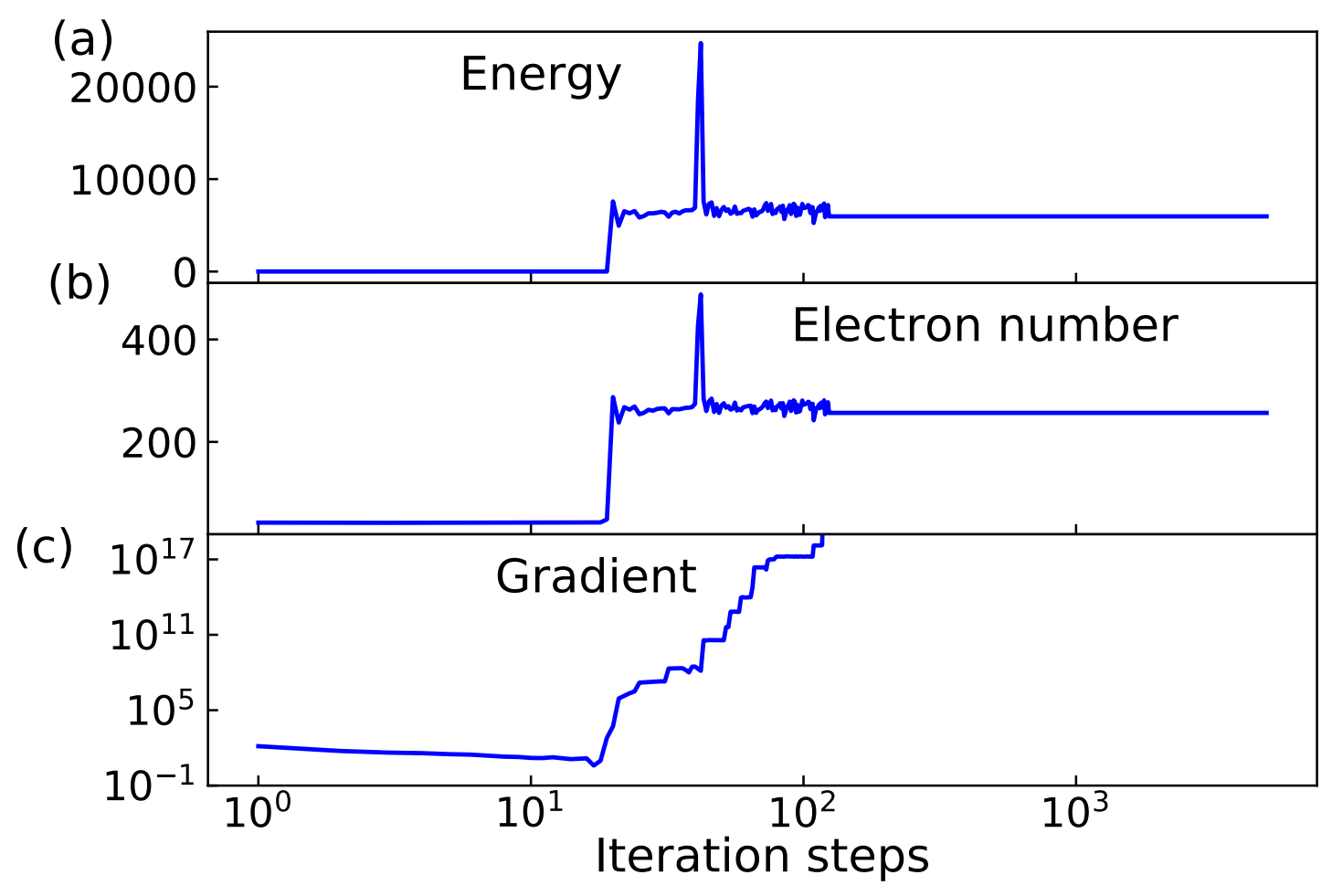

Figure S3: Failure of LM in the treatment of the ensemble N-representability constraint. $\mathrm{C}_{6} \mathrm{H}_{6}$ is calculated, and the changes of (a) energy, (b) total electron number, and (c) gradient are plotted for each step during occupation optimization. The power functional with $\omega=0.5$ is applied. The optimal energy by EBI is set to zero for (a). All the data are in au. 


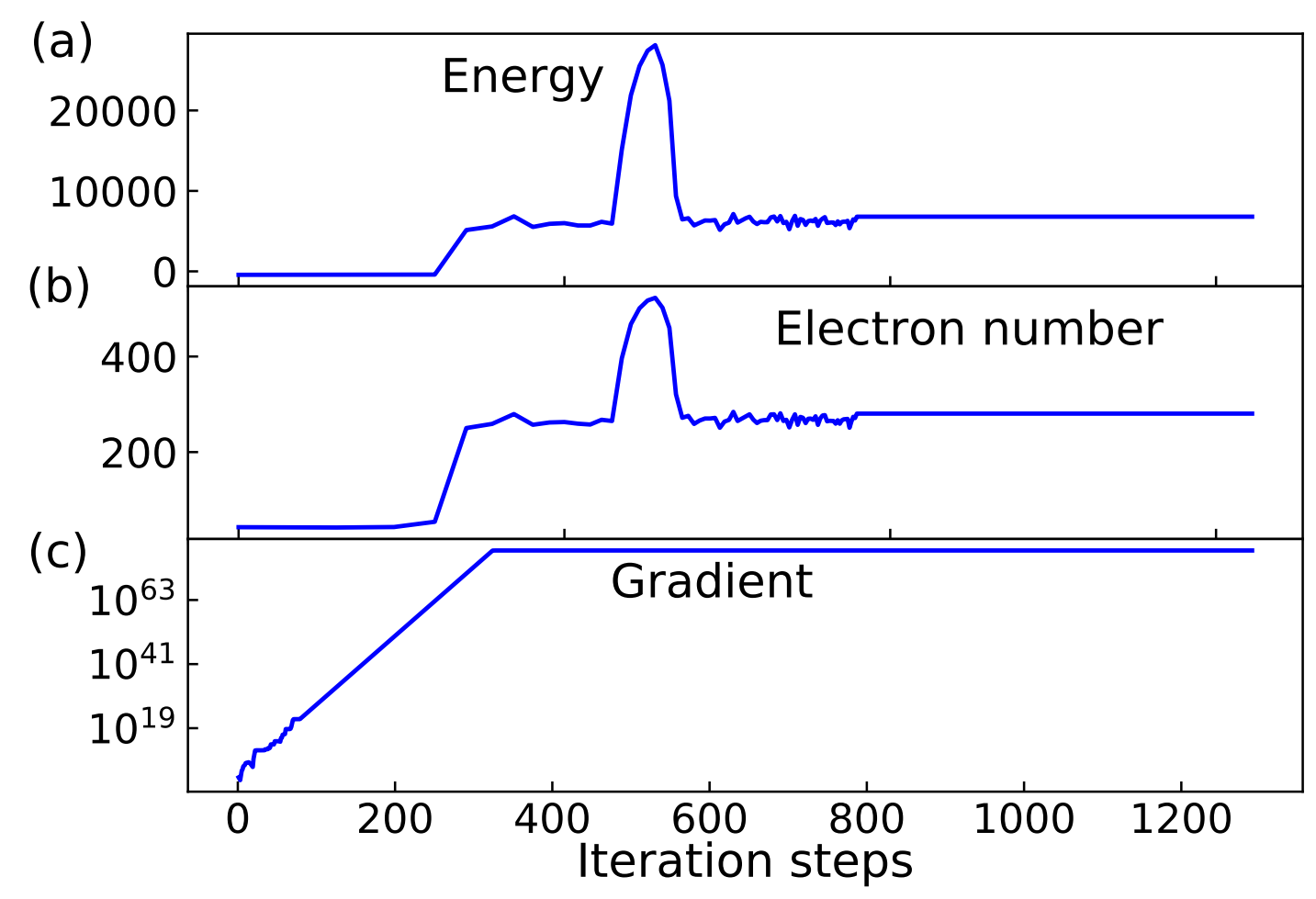

Figure S4: Failure of LM in the treatment of the ensemble N-representability constraint. $\mathrm{C}_{6} \mathrm{H}_{6}$ is calculated, and the changes of (a) energy, (b) total electron number, and (c) gradient are plotted for each step during occupation optimization. The power functional with $\omega=0.5$ is applied. The optimal energy by EBI is set to zero for (a). All the data are in au. 


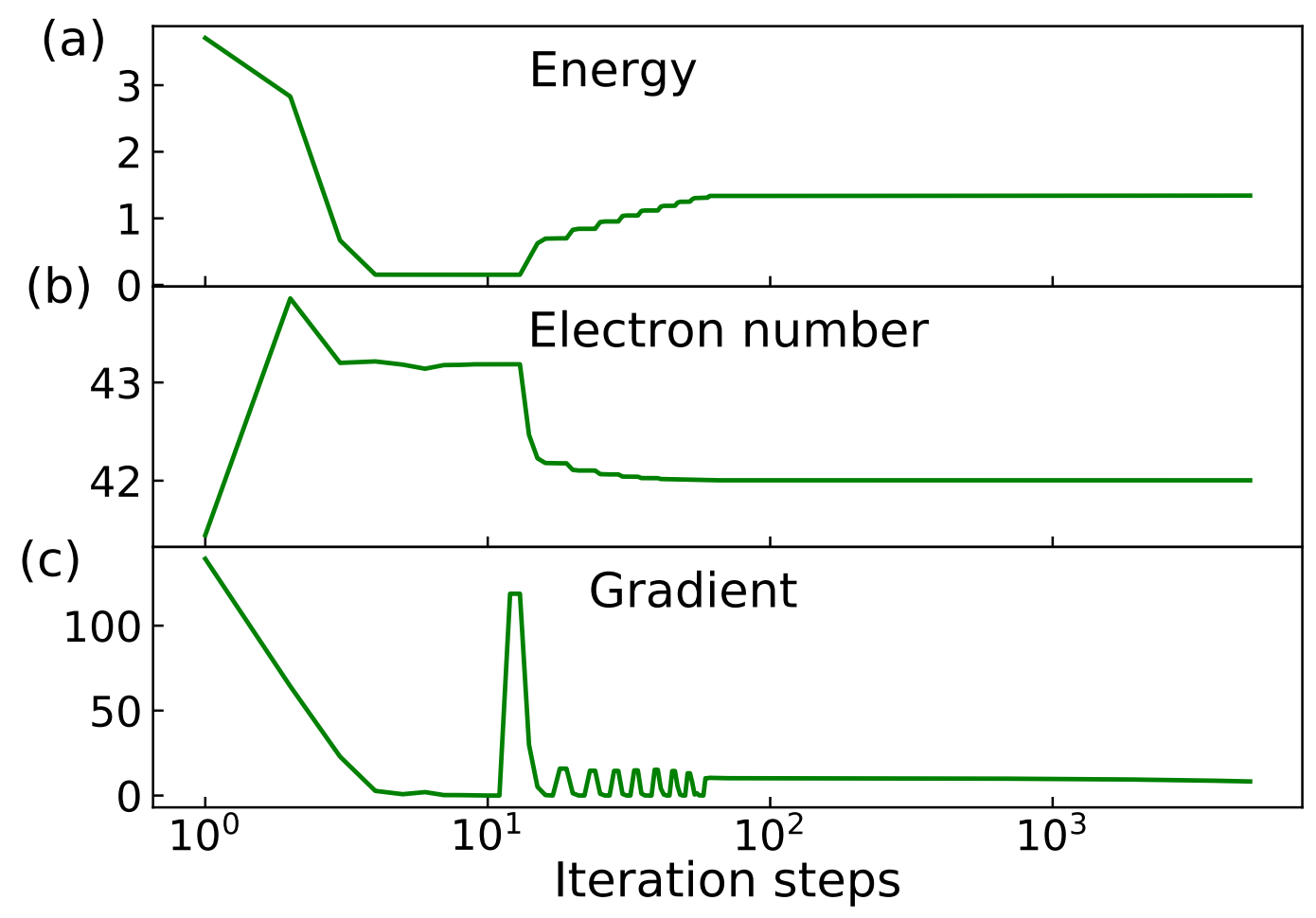

Figure S5: Failure of ALM in the treatment of the ensemble N-representability constraint. $\mathrm{C}_{6} \mathrm{H}_{6}$ is calculated, and the changes of (a) energy, (b) total electron number, and (c) gradient are plotted for each step during occupation optimization. The power functional with $\omega=0.5$ is applied. The optimal energy by EBI is set to zero for (a). All the data are in au.

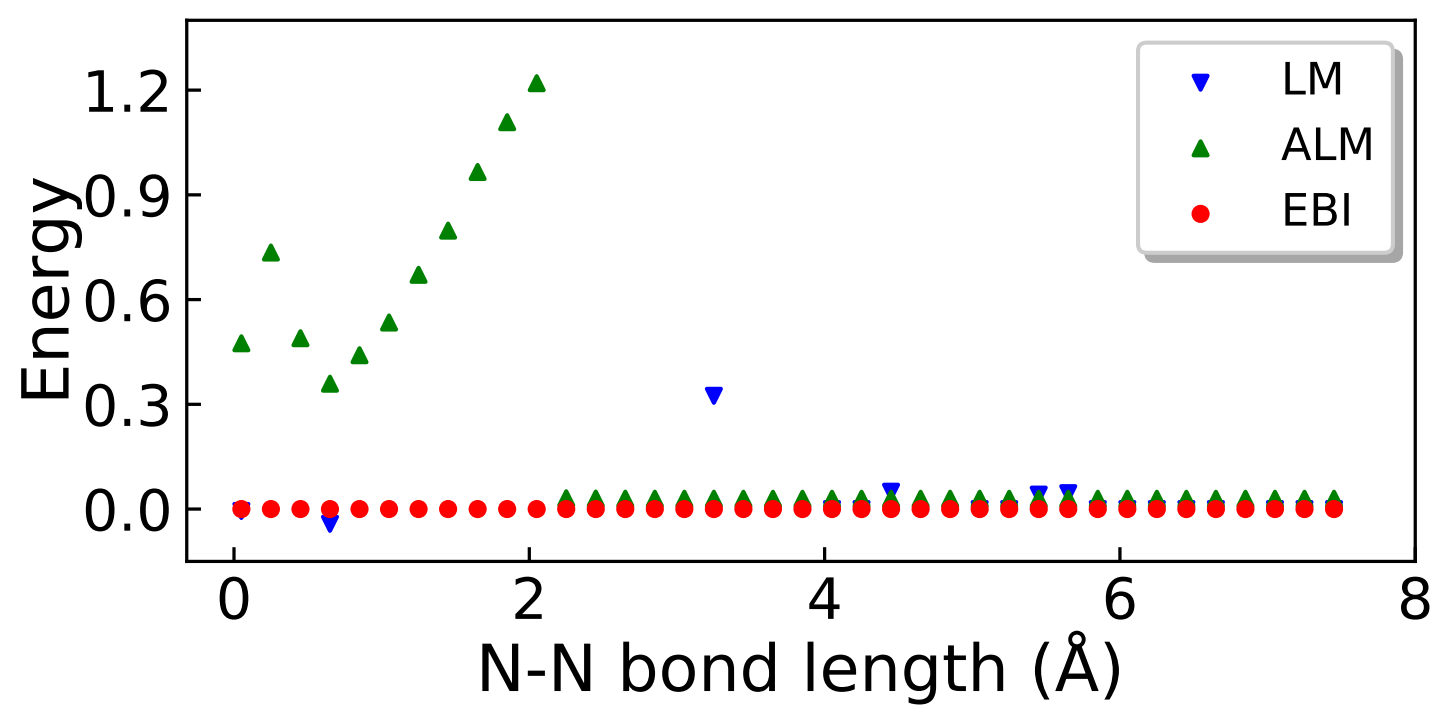

Figure S6: Constrained optimization for energies of $\mathrm{N}_{2}$ with increasing bond lengths. Energies of the power functional with $\omega=0.5$ are plotted after natural occupation optimization by LM, ALM, and EBI, the optimal energies by EBI are set to zero. The same initial guesses of occupations are used for LM, ALM, and EBI. All energies are in au. 

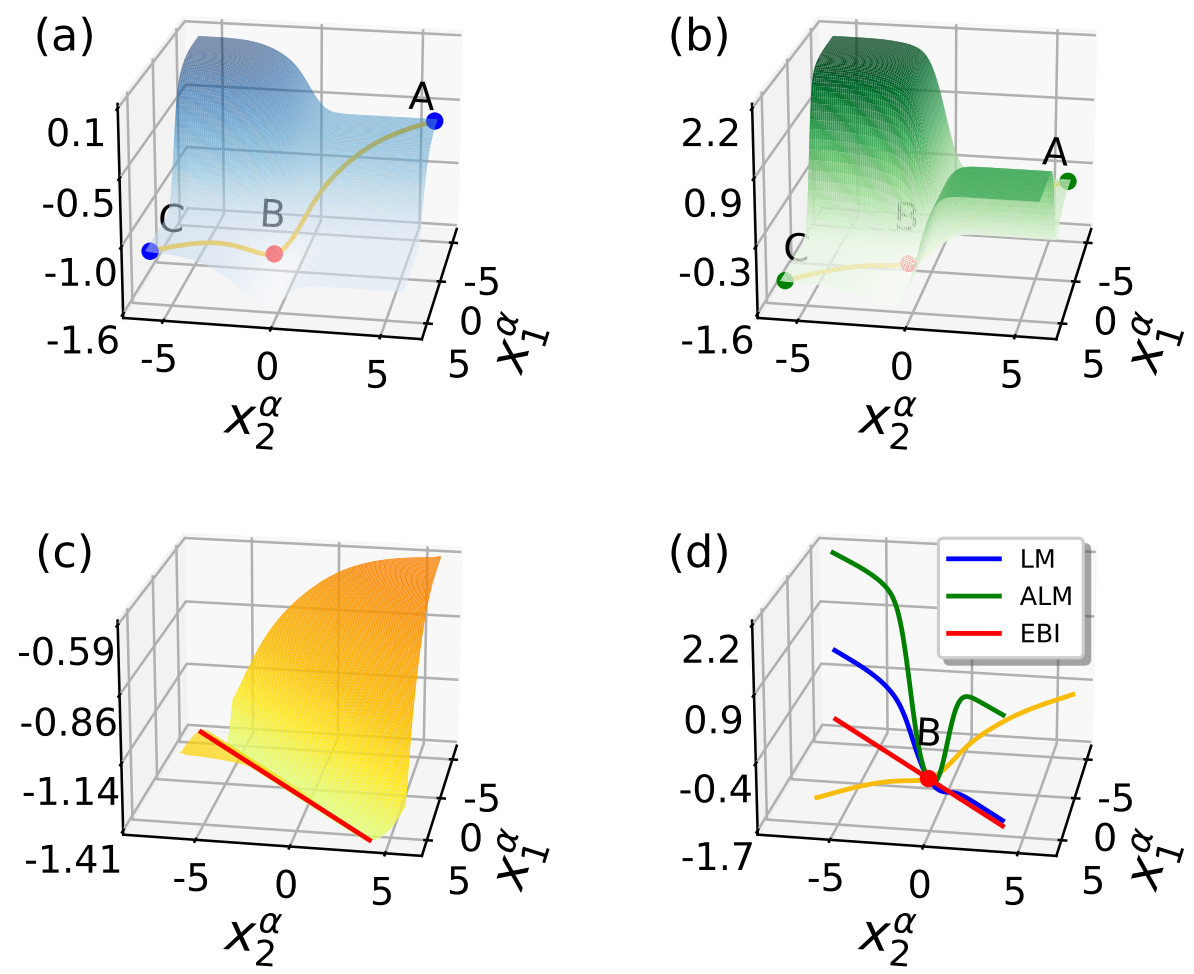

Figure S7: Objective functions for $\mathrm{H}_{2}^{+}$with the minimal basis set in LM, ALM, and EBI. The fermionic constraint is considered via the error function, i.e. Eq. (S24) for EBI and Eq. (S24) without the implicit function $\mu^{\sigma}$ for both LM and ALM. They are (a) the Lagrangian of Eq. S7 with $\lambda^{\alpha}=-0.03$; (b) the augmented Lagrangian of Eq. S19 with $\lambda_{k}^{\alpha}=-0.03$ and $c_{k}^{\alpha}=4$; (c) the electronic energy as an implicit function of $\left(x_{1}^{\alpha}, x_{2}^{\alpha}\right)$; and (d) the objective functions of LM with $\lambda^{\alpha}=-0.03$ (blue), ALM with $\lambda_{k}^{\alpha}=-0.03$ and $c_{k}^{\alpha}=4$ (green), and EBI (red). A, B, and C in (a)-(d) are critical points. The points that satisfy the particle number conservation constraint (i.e. Eq. S3) are in yellow, and the optimal solutions are in red. Hence, the points that satisfy Eq. S3 form a curve in LM and ALM, and the optimal solution is a point (i.e. $B$ ) in the curve. In contrast, all points satisfy Eq. S5 in EBI, and the optimal solutions are a line (all points in the line giving the same occupations and energy). The power functional with $\omega=0.5$ is applied. All the data are in au. 


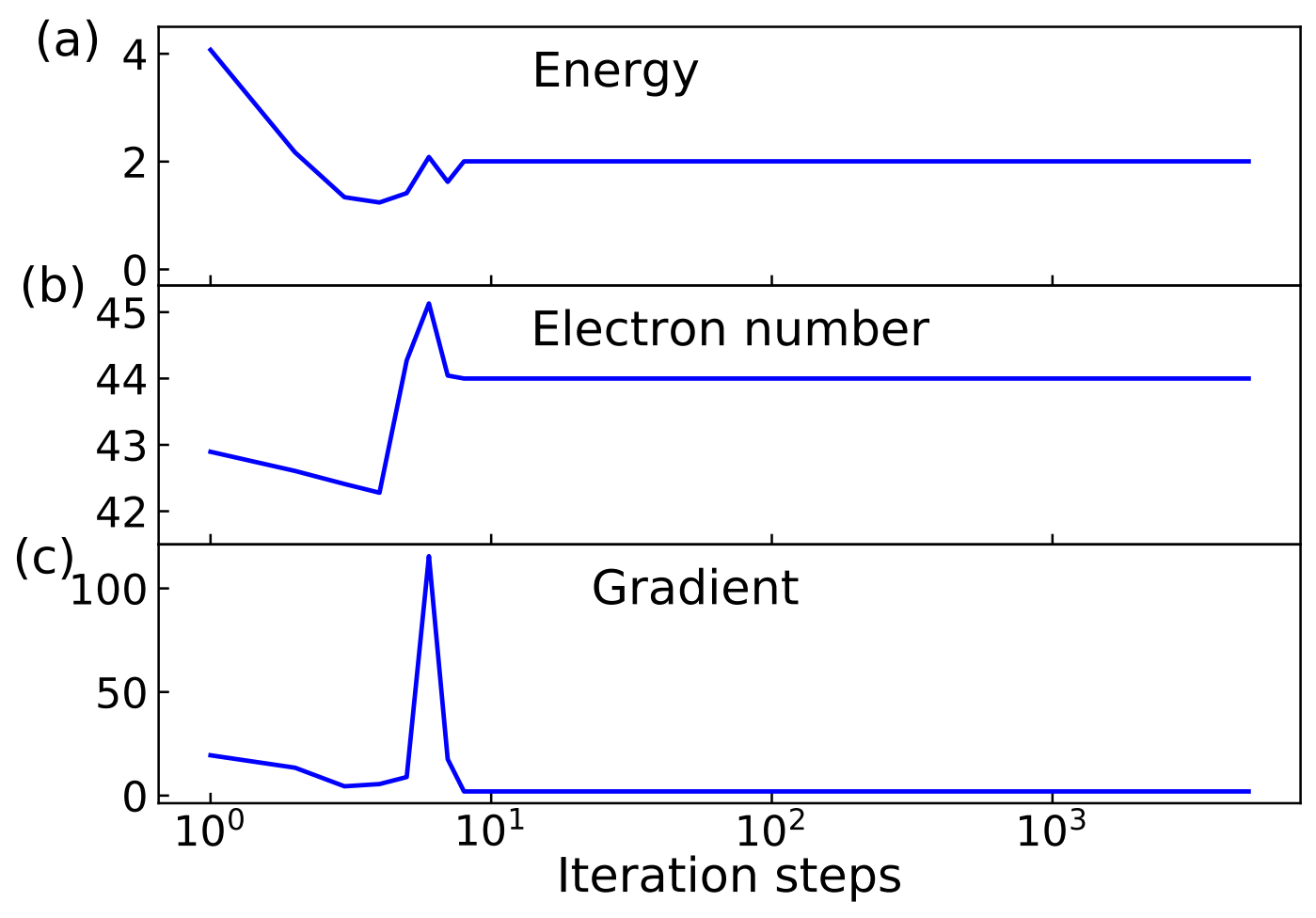

Figure S8: Failure of LM in the treatment of the ensemble N-representability constraint. The fermionic constraint is considered via the error function, i.e. Eq. (S24) without the implicit function $\mu^{\sigma} \cdot \mathrm{C}_{6} \mathrm{H}_{6}$ is calculated, and the changes of (a) energy, (b) total electron number, and (c) gradient are plotted for each step during occupation optimization. The power functional with $\omega=0.5$ is applied. The optimal energy by EBI is set to zero for (a). All the data are in au. 


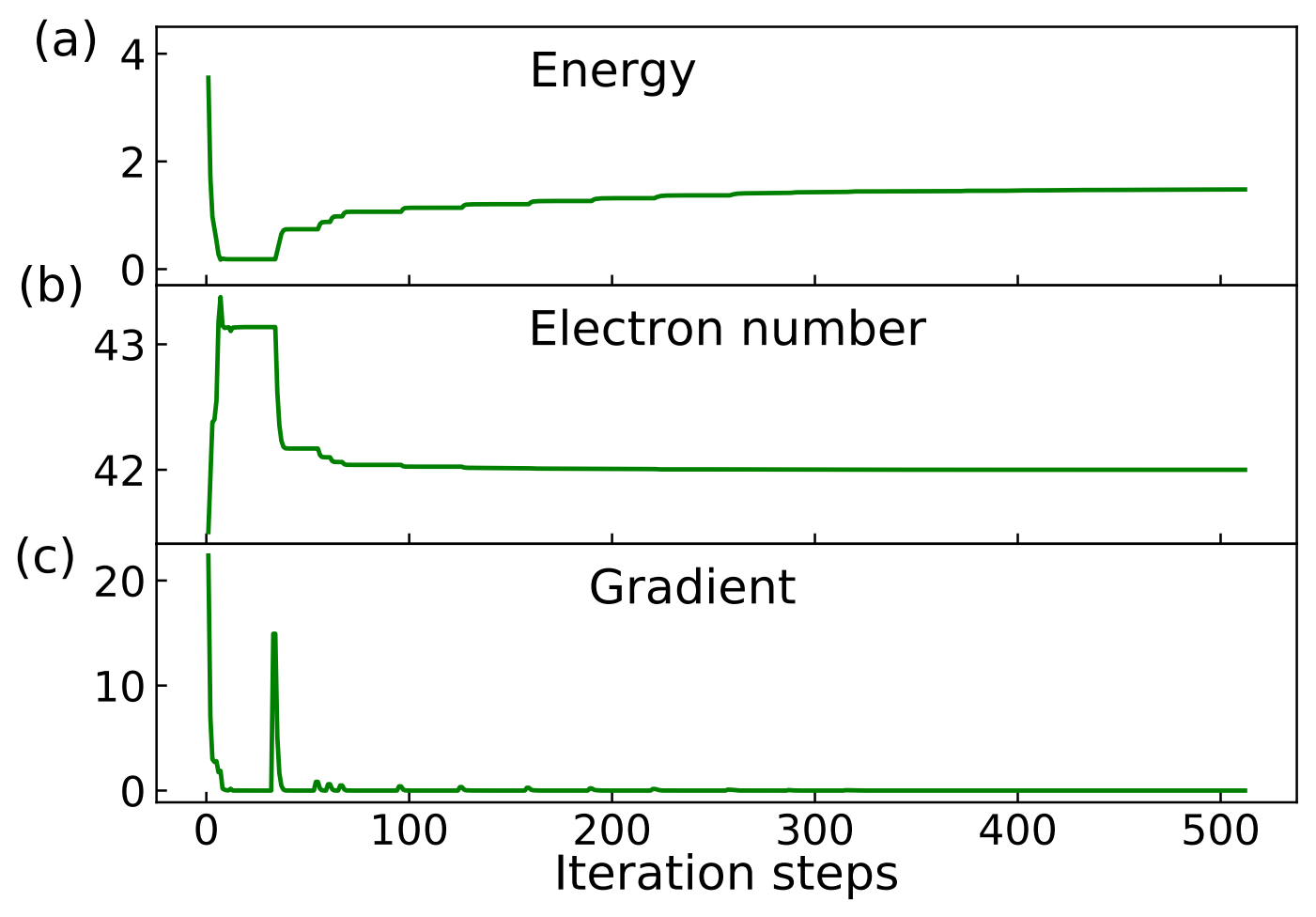

Figure S9: Failure of ALM in the treatment of the ensemble N-representability constraint. The fermionic constraint is considered via the error function, i.e. Eq. (S24) without the implicit function $\mu^{\sigma} \cdot \mathrm{C}_{6} \mathrm{H}_{6}$ is calculated, and the changes of (a) energy, (b) total electron number, and (c) gradient are plotted for each step during occupation optimization. The power functional with $\omega=0.5$ is applied. The optimal energy by EBI is set to zero for (a). All the data are in au. 


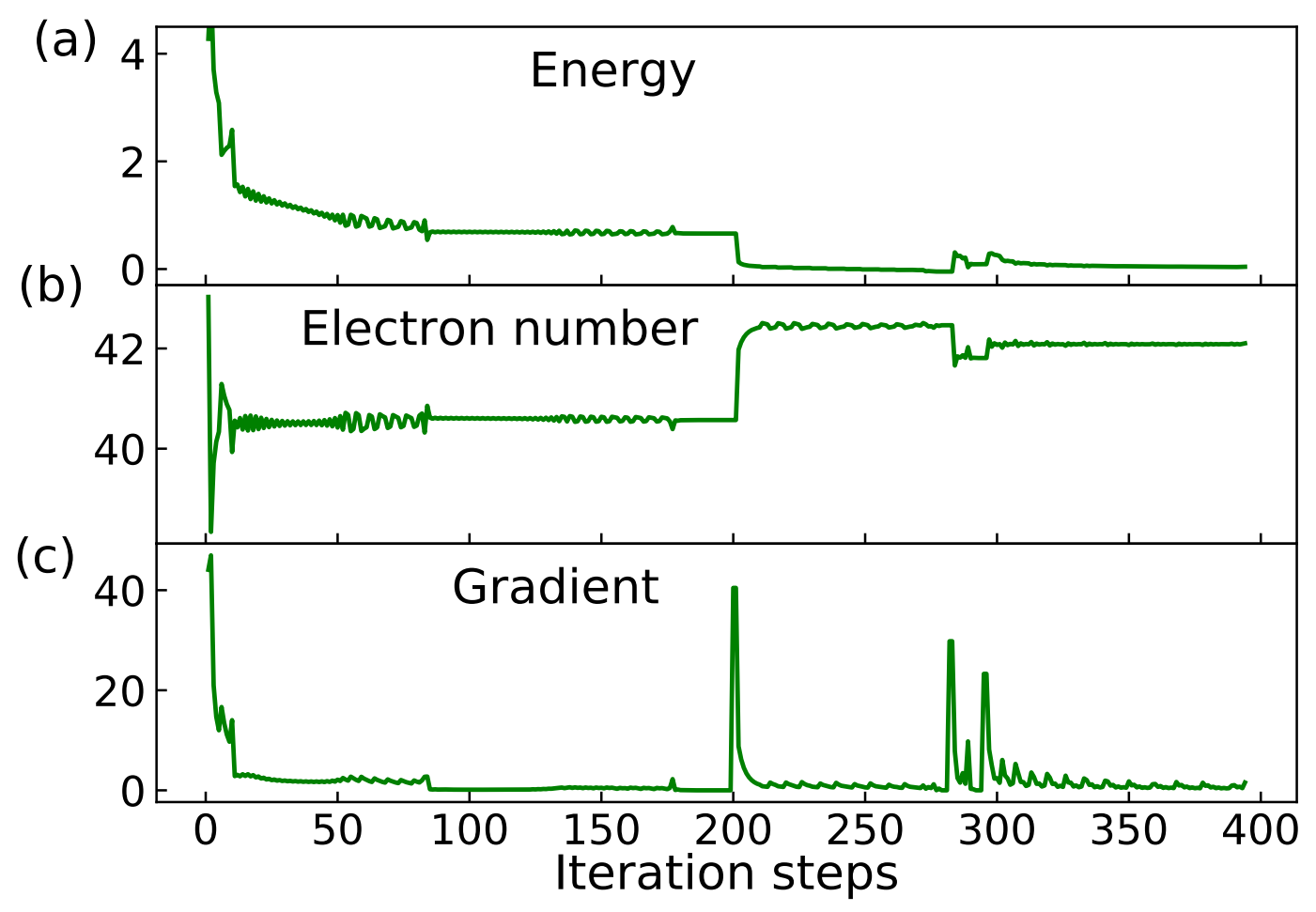

Figure S10: Failure of ALM in the treatment of the ensemble N-representability constraint. The fermionic constraint is considered via the error function, i.e. Eq. (S24) without the implicit function $\mu^{\sigma} \cdot \mathrm{C}_{6} \mathrm{H}_{6}$ is calculated, and the changes of (a) energy, (b) total electron number, and (c) gradient are plotted for each step during occupation optimization. The power functional with $\omega=0.5$ is applied. The optimal energy by EBI is set to zero for (a). All the data are in au. 


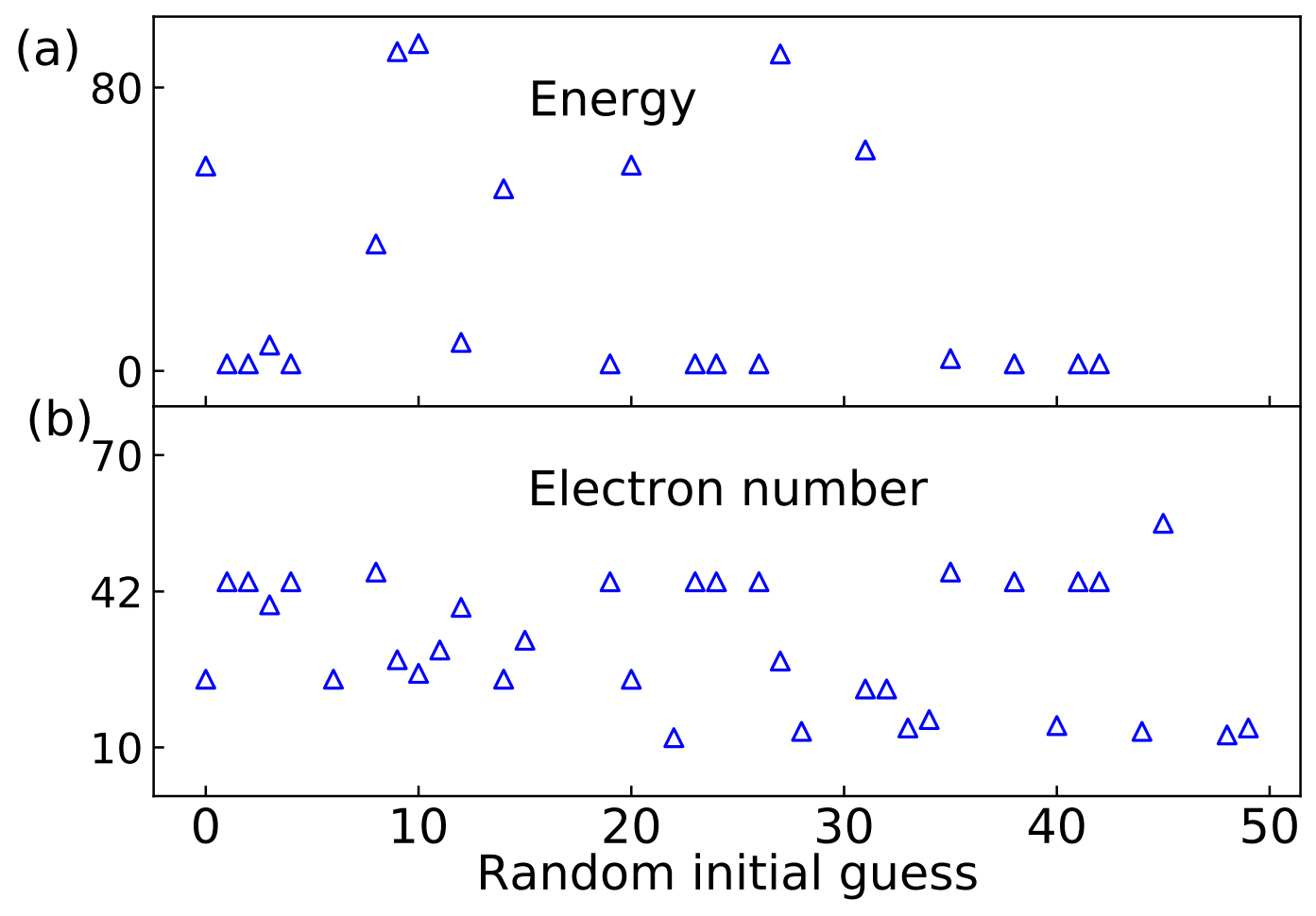

Figure S11: Natural occupation optimization for $\mathrm{C}_{6} \mathrm{H}_{6}$ in LM. The fermionic constraint is considered via the error function, i.e. Eq. (S24) without the implicit function $\mu^{\sigma}$. The resulting (a) energies and (b) total electron numbers with 50 different random initial guesses are plotted. The power functional with $\omega=0.5$ is applied. The optimal energy by EBI is set to zero for (a). All the data are in au. 

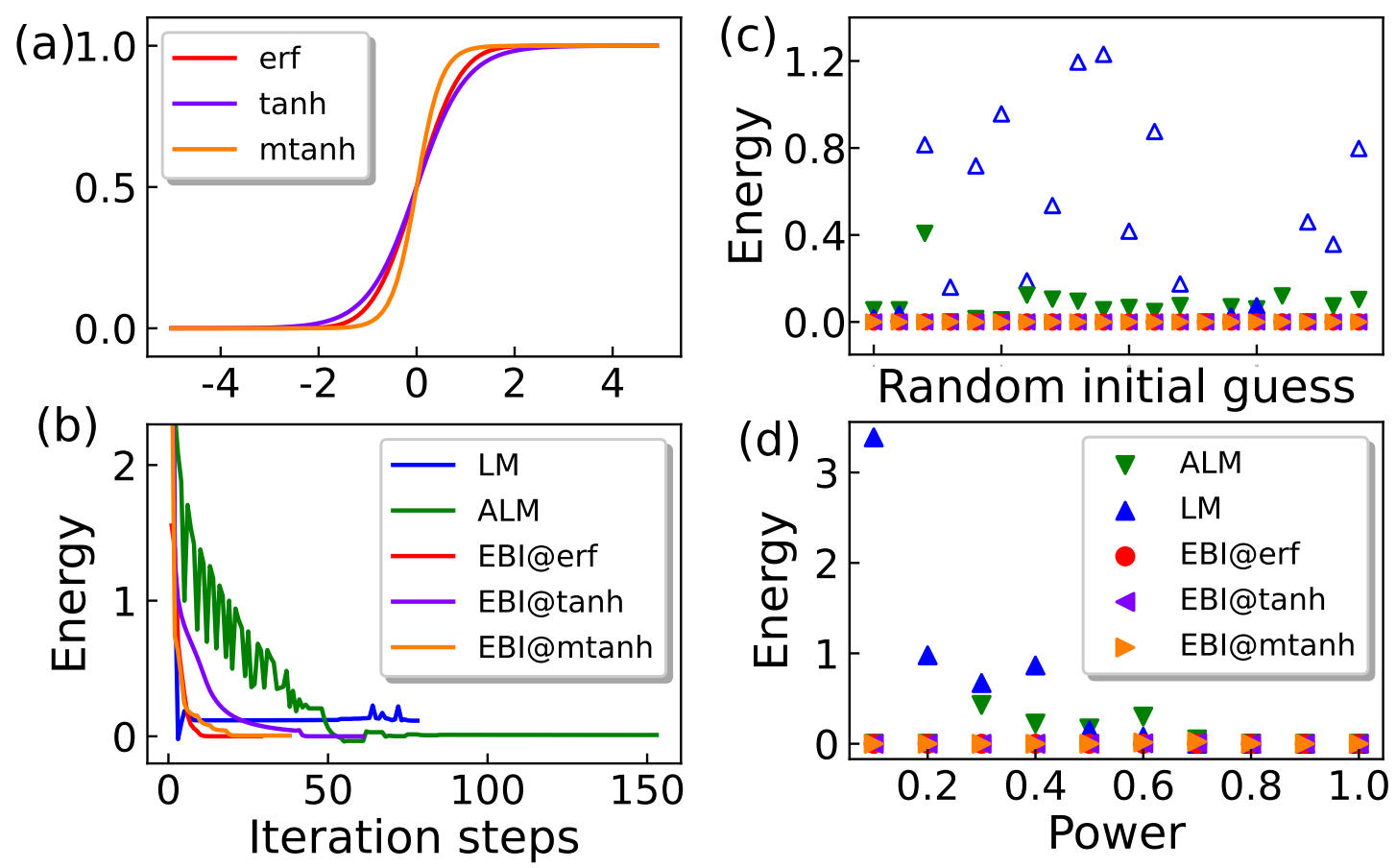

Figure S12: Comparison between different sigmoid functions for natural occupations in EBI. (a) Three sigmoid functions (i.e. Eqs. S24, S42, and S43) are checked, which invoke error function (erf), hyperbolic tangent function (tanh), and modified hyperbolic tangent function (mtanh) respectively. Natural occupation optimization for $\mathrm{C}_{6} \mathrm{H}_{6}$ is calculated, and the performances of different methods are evaluated by (b) the change of energy for each step during optimization in use of the power functional with $\omega=0.5$; and by converged energies for (c) 20 randomly generated initial guesses and for $(\mathrm{d})$ the power functionals with $\omega$ ranging from 0.1 to 1.0. The optimal energy by EBI@erf is set to zero for (b), (c), and (d). The test results of LM and ALM are also included for better comparison. Solid and hollow symbols in (c) indicate the final recorded energies are convergent and non-convergent respectively. All the data are in au. 


\section{References}

(1) Gilbert, T. L. Hohenberg-Kohn Theorem for Nonlocal External Potentials. Phys. Rev. B 1975, 12, 2111-2120.

(2) Levy, M. Universal Variational Functionals of Electron Densities, First-Order Density Matrices, and Natural Spin-Orbitals and Solution of the v-Representability Problem. Proc. Natl. Acad. Sci. USA 1979, 76, 6062-6065.

(3) Löwdin, P.-O. Quantum Theory of Many-Particle Systems. I. Physical Interpretations by Means of Density Matrices, Natural Spin-Orbitals, and Convergence Problems in the Method of Configurational Interaction. Phys. Rev. 1955, 97, 1474-1489.

(4) Coleman, A. J. Structure of Fermion Density Matrices. Rev. Mod. Phys. 1963, 35, 668686.

(5) Sharma, S.; Dewhurst, J. K.; Lathiotakis, N. N.; Gross, E. K. U. Reduced Density Matrix Functional for Many-Electron Systems. Phys. Rev. B 2008, 78, 201103(R).

(6) Bertsekas, D. P. Constrained Optimization and Lagrange Multiplier Methods; Academic press, 2014.

(7) Aprá, E. et al. NWChem: Past, Present, and Future. J. Chem. Phys. 2020, 152, 184102.

(8) Dunning, T. H. Gaussian Basis Sets for Use in Correlated Molecular Calculations. I. The Atoms Boron Through Neon and Hydrogen. J. Chem. Phys. 1989, 90, 1007-1023.

(9) Kendall, R. A.; Dunning, T. H.; Harrison, R. J. Electron Affinities of the First-Row Atoms Revisited. Systematic Basis Sets and Wave Functions. J. Chem. Phys. 1992, 96, 6796-6806. 